\title{
Exceptional Cause of Appendicular Peritonite: Foreign Metal Object in Appendicular Lumen About A Case
}

Christel Marie Lalèyè , Setondji Gilles Roger Attolou, Félix Kamga, Elohonnan Wilfried Gandji, Yakoubou Imorou, Habib N'Domè Natta N’tcha, Woléola Pencôme Ogouyemi, Kuassi Delphin Mehinto

"'Hubert KoutouKou Maga", University Teaching Hospital Center, Cotonou, Bénin

DOI: $10.36347 /$ sasjs.2020.v06i04.012

| Received: 19.04.2020 | Accepted: 27.04.2020 | Published: 30.04.2020

*Corresponding author: Christel Marie Lalèyè

Abstract

Case Report

Introduction: Acute appendicitis is rarely caused by the obstruction of the appendicular lumen by an intraluminal metal object. Clinical Case: Patient is 24 year old woman with no prévious history admitted for generalized abdominal pain starting at the right iliac fossa (RIF) for about 07 days, associated with vomiting, fever and transit stop. The clinical examination reveal a syndrome of peritoneal irritation. The diagnosis of peritonitis of appendicular origin was made and the patient operated. Peroperatively, we discoved false membranes, dilated and agglutinated small intestine in the right iliac fossa. The appendix was unperforated and phlegmonous. An appendectomy was performed followed by washing and drainage of the abdominal cavity. At the opening of the appendix we discoved a metal clip obstructing the appendicular lumen. Discussion: In the etiopathogenesis of acute appendicitis, it is classically described that it is an obstruction of the appendicular lumen trapping bacteria which is at the origin of the onset of symptoms. Intraappendicular foreign bodies have been described as dental amalgam, pin, nails, or staples.

Keywords: appendicitis, peritonitis, foreign object, metal.

Copyright @ 2020: This is an open-access article distributed under the terms of the Creative Commons Attribution license which permits unrestricted use, distribution, and reproduction in any medium for non-commercial use (NonCommercial, or CC-BY-NC) provided the original author and source are credited.

\section{INTRODUCTION}

Appendicitis is an acute inflammation of the ileocaecal appendix. One of the mechanisms involved in its occurrence is that of the obstruction of the appendicular lumen [1,2]. Usually this obstruction is carried out by a stercolite but in some cases it caused by obstruction by a metallic object. We report the case of appendicitis complicated by peritonitis with the presence of a metal staple in the appendicular lumen, with review of the literature.

\section{CLINICAL CASE}

24 year old patient with no previous history. Referral from a local clinic for a suspicion of generalized acute peritonitis of appendicular origin. The clinical examination noted:

- A start going back to 07 days;

- Pain starting at the right iliac fossa (RIF) secondarily generalized to the entire abdomen;

- Patient had nausea, fever, vomiting and transit stop.

The physical examination noted: a good general condition; peritoneal irritation syndrome, with maximum pain in the right iliac fossa.
An x-ray of the abdomen without preparation reveald a diffuse greyness; the hydro-aeric images of the small intestine and an opacity of the size of a rice grain in the RIF (Figure-1). The diagnosis of appendicular peritonitis was made, hence the operative indication.

In the operating room; under general anesthesia, after midline incision straddling the umbilicus, peritoneal fluid and false membranes were discovered; small intestine dilated and agglutinated in the RIF around the appendix. The release allowed us to discover:

- A small parieto-bridle flange with a junctional level about $20 \mathrm{~cm}$ from the ileo-caecal crossroads (Figures 2 and 3);

- A phlegmonous appendix with false membrane.

An appendectomy and abdominal toilet were performed, followed by drying and closing plan by plan. The opening of the appendix part allowed us to discover a staple in the lumen at the end of the appendix without stercolites (Figure-4). The post-operative 
follow-ups were simple; the patient was discharged afterrr the 6 th post-operative day.

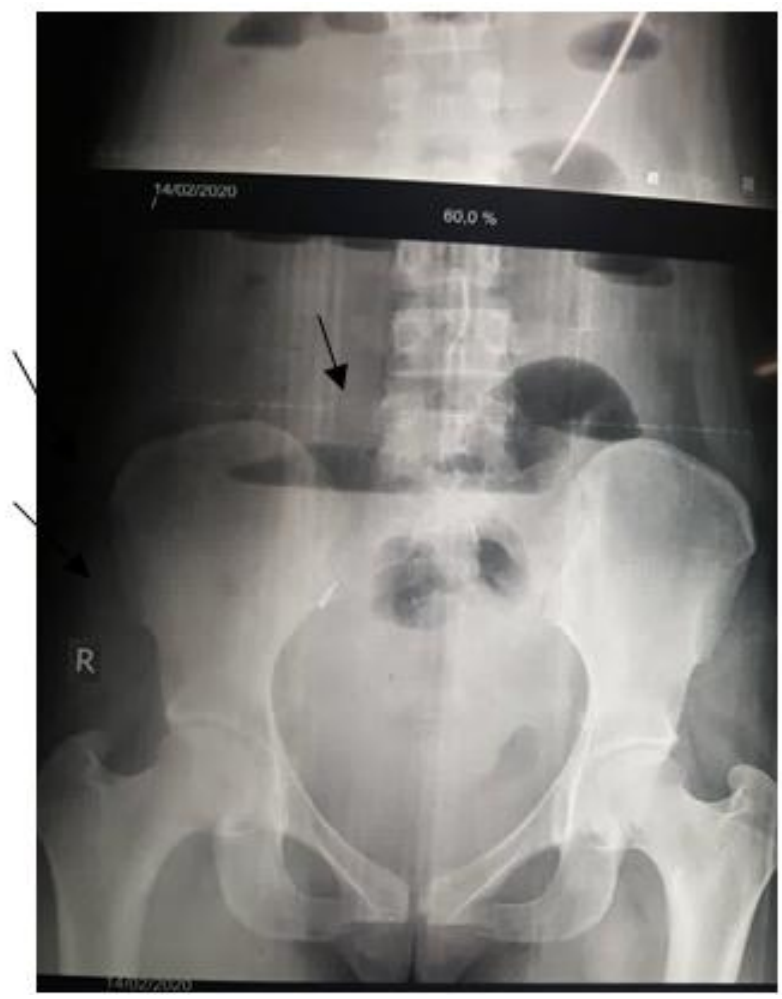

Fig-1: Hydroaeric images of the small intestine and radiopaque foreign object in RIF

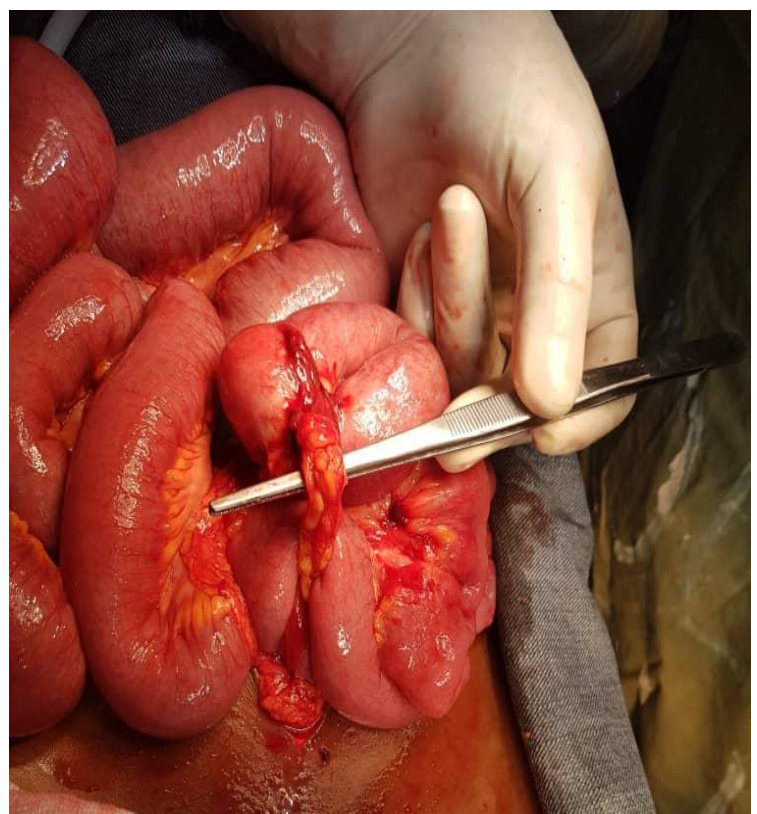

Fig-2: Epiploo-parietal flange cravating the small intestine

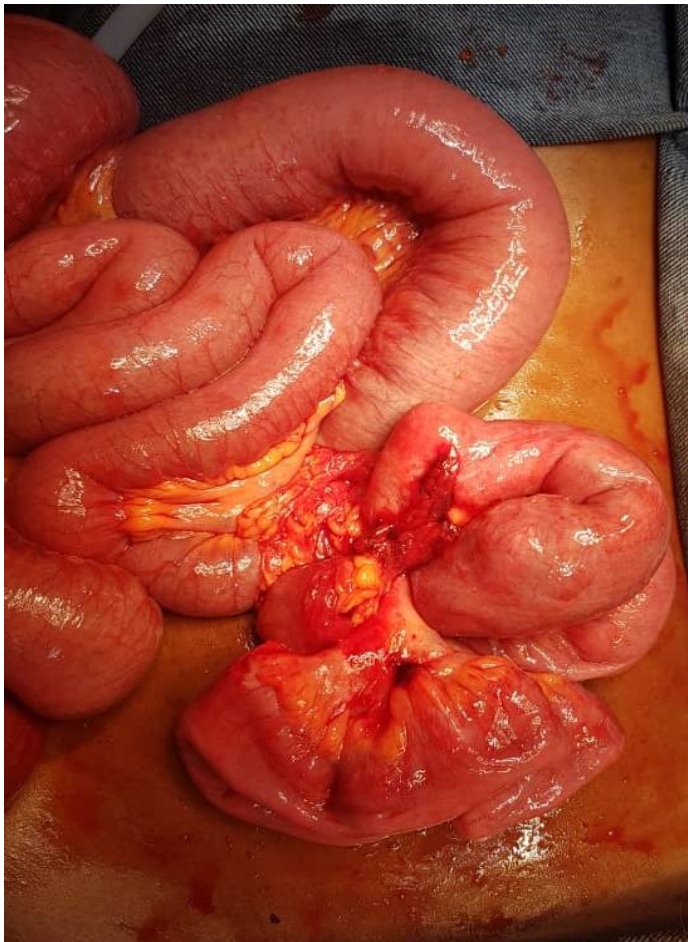

Fig-3: After section of the flange, highlighting a junctional level

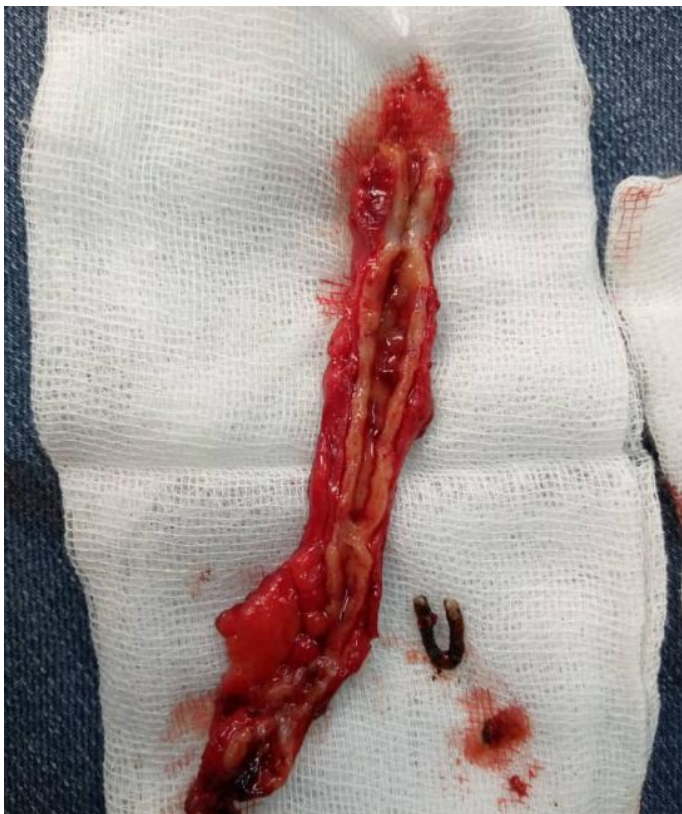

Fig-4: Opening of the appendix, discovery of a metallic foreign object

\section{DISCUSSION}

Many authors in the literature have described intra-appendicular foreign objects; these may be dental amalgam, pin, nails, or sinkers or staples [3, 4]. They are generally radio opaque $[4,5]$. In some cases, the notion of ingestion of these foreign objects has been found in other countries. In the mechanism of occurrence of acute appendicitis it is classically described as an obstruction of the appendicular lumen trapping bacteria inside which is generally at the origin of the occurrence of the symptomatology [6]. 
However, studies have revealed that an intraappendicular foreign objects with a pointed tip or a sharp edge, even if it does not obstruct the lumen, can be at the origin of the occurrence of acute appendicitis, [3] this is due to the the point of the foreign body would irritate the appendicular mucosa, thus causing an inflammatory reaction which causes acute appendicitis [7].

E. Delabrousse \& al, accidentally discovered several intra-appendicular pellets in a 70-year-old man without any sign of acute appendicitis. They explained the absence of appendicular symptomatology by the fact that the pellets were not obstructive and were rounded in shape [8].

In our clinical case, it was a complication of acute appendicitis, a type of peritonitis with reflex intestinal obstruction. There was no perforation of the appendix but false membranes at its end, and in the lumen was discovered a staple with two legs with pointed ends, and a rounded end embedded in the appendix tip. We can therefore question about the obstructive nature of the clip and not its pointed ends, giving that there is no perforation of the appendix.

A similar case was described by MEHINTO \& al where an obstructive metallic intra-appendicular foreign object was discovered in the lumen after an appendectomy, which is indicated for acute appendicitis [9]. Our case corroborates numerous studies in which the intra-appendicular foreign bodies which were symptomatic had between 70 and $90 \%$ a pointed tip, or a sharp edge [7]. An appendectomy is therefore recommended as soon as the foreign body present in the appendix runs the risk of acute appendicitis or appendicular perforation $[3,10]$.

\section{CONCLUSION}

Intra-appendicular foreign objects are most often symptomatic if they have a sharp tip or a sharp edge, exposing them to the risk of appendicular perforation. When the preoperative discovery is made, even in the absence of signs of acute appendicitis, an appendectomy must be systematic. The peculiarity of our case lies in the clinical presentation which is that of a complication of appendicitis. The discovery of the metal object is therefore not accidental because the appendix is already incriminated. Rather, it is a rare cause of appendicitis complication.

\section{REFERENCES}

1. Jacobs DO. Acute appendicitis and peritonitis. Dans Harrison's Principles of Internal Medecine. Edition McGraw-Hill Professional. 2015. Chapter 356.

2. Arnbjörnsson E, Bengmark S. Obstruction of the appendix lumen in relation to pathogenesis of acute appendicitis. Acta Chir Scand. 1983; 149: 789-91.

3. Mellor TK, Mellor SG. Forein bodies of dental origin in the appendix. JR armymed corps, 1995;141:174-6.

4. Klingler P, Seelig M, DeVault K, Wetscher G, Floch N, Branton S, Hinder R. Ingested foreign bodies within the appendix: a 100-year review of the literature. Digestive Diseases. 1998;16(5):30814.

5. Matthias J, Bruot O, Ganne PA, Laurent V, Regent D. Appendicite. Dans Radiodiagnostic- Appareil digestif. Encycl Méd Chir Edition Elsevier Masson. 2008, 33-472-G-10.

6. Borie F, Philippe C. Appendicite aiguë. EMC (Elsevier Masson SAS, Paris), Gastro-entérologie, 2011; 9-066-A-10.

7. Balch CM. SILVERD. Foreign bodies in the appendix. ilrchSf/rg, 1971;102:14-20.

8. Delabrousse E. Plombs de chasse intra appendiculaire Journal Radiol, 2002;83:169-7.

9. Mehinto DK, Bagnan OK, Padonou N. Appendicite aigue par obstruction de l'appendice par un métal intra luminal de provenance non élucidée. Médecine d'Afrique noir, 2010;5710: 445-447.

10. Deffrene P, Pracros JP. Les corps étrangers intraappendiculaires. JBR BTR, 1987;70 :205-9. 